\title{
Using ADOPT Algorithm and Operational Data to Discover Precursors to Aviation Adverse Events
}

\author{
Vijay Janakiraman, Bryan Matthews and Nikunj Oza \\ Data Sciences Group \\ NASA Ames Research Center, CA, USA
}

AIAA Science and Technology Forum and Exposition (SciTech 2018)

Session: IS-15, Learning, Reasoning, and Data-Driven Systems I 


\section{Outline}

- Background

- Precursor discovery problem, uses, challenges

- Methodology

- ADOPT algorithm

- Case Studies

- Take-off Stall Hazard

- STAR procedure adherence

- Summary 


\section{Outline}

- Background

- Precursor discovery problem, uses, challenges

- Methodology

- ADOPT algorithm

- Case Studies

- Take-off Stall Hazard

- STAR procedure adherence

- Summary 
Precursor discovery 


\section{Precursor discovery}

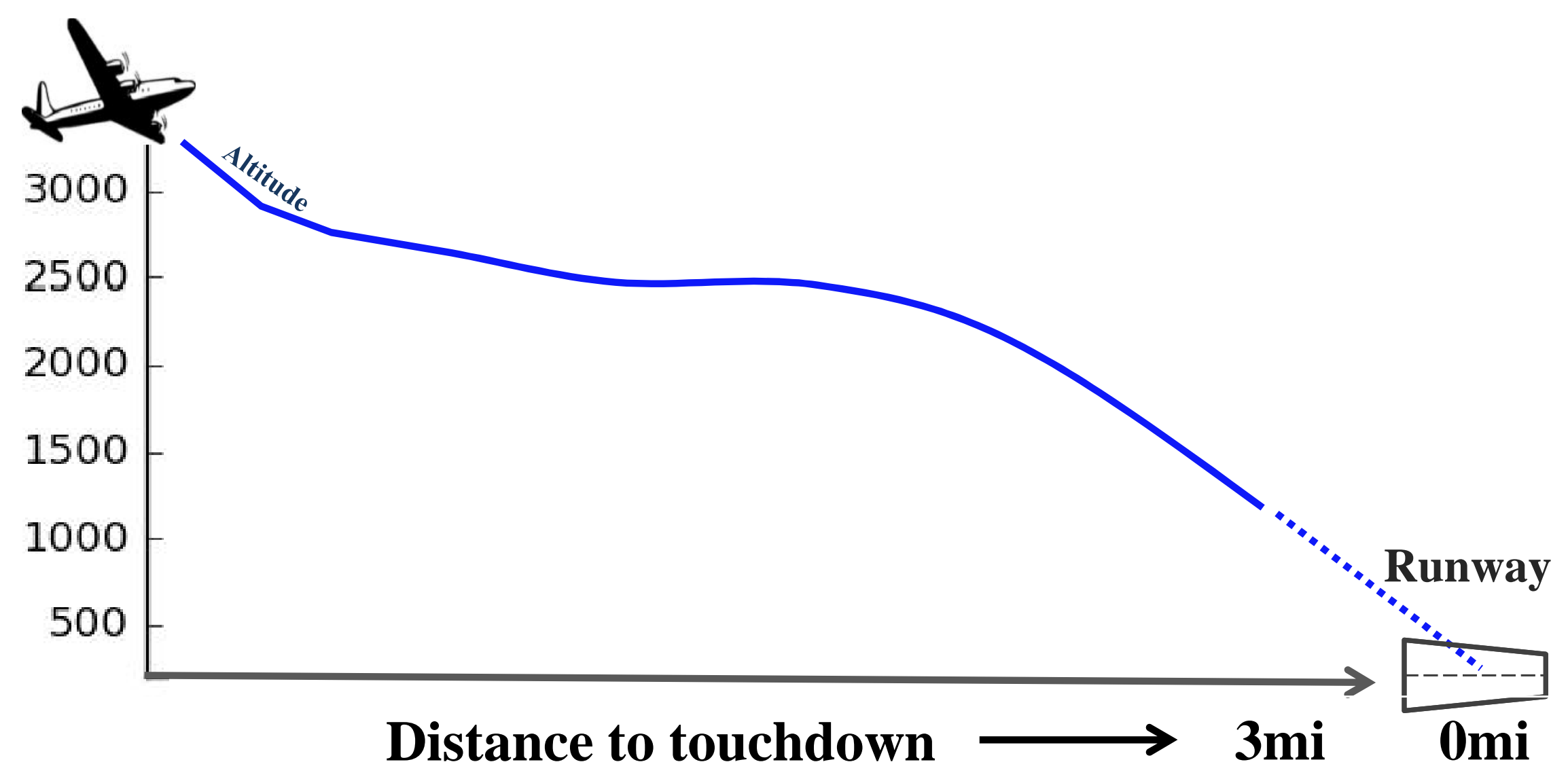




\section{Precursor discovery}

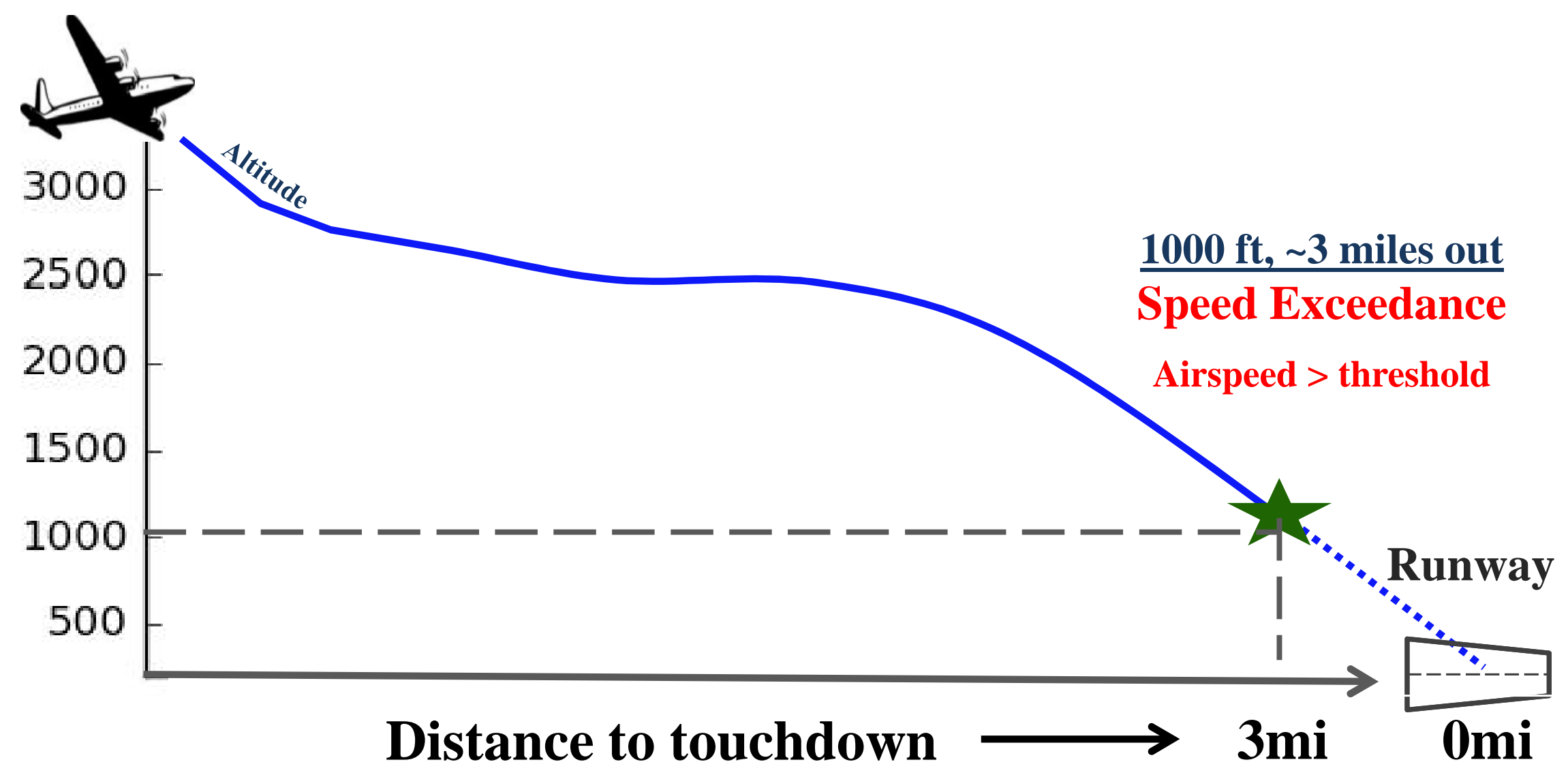




\section{Precursor discovery}

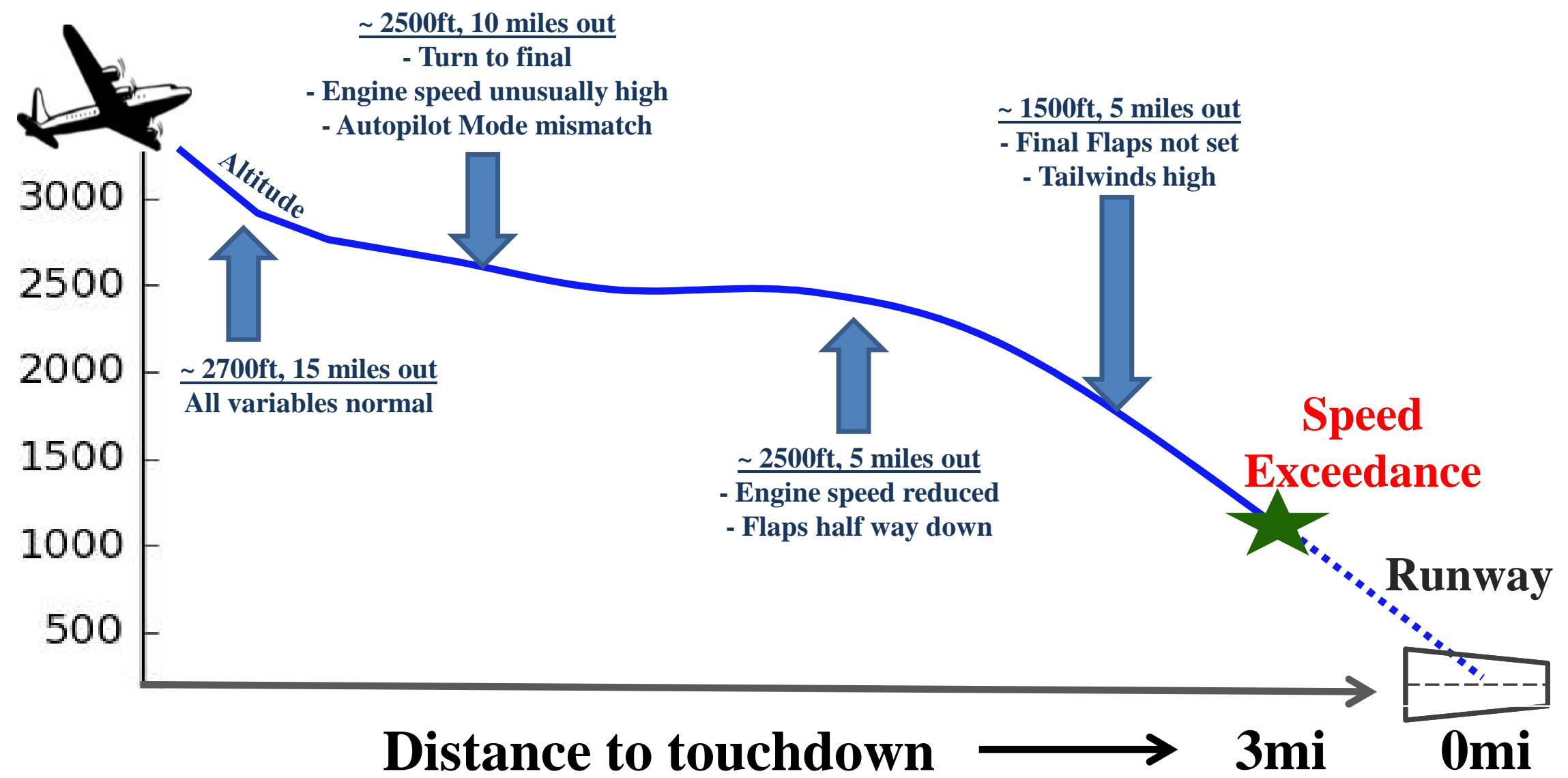




\section{Precursor discovery}

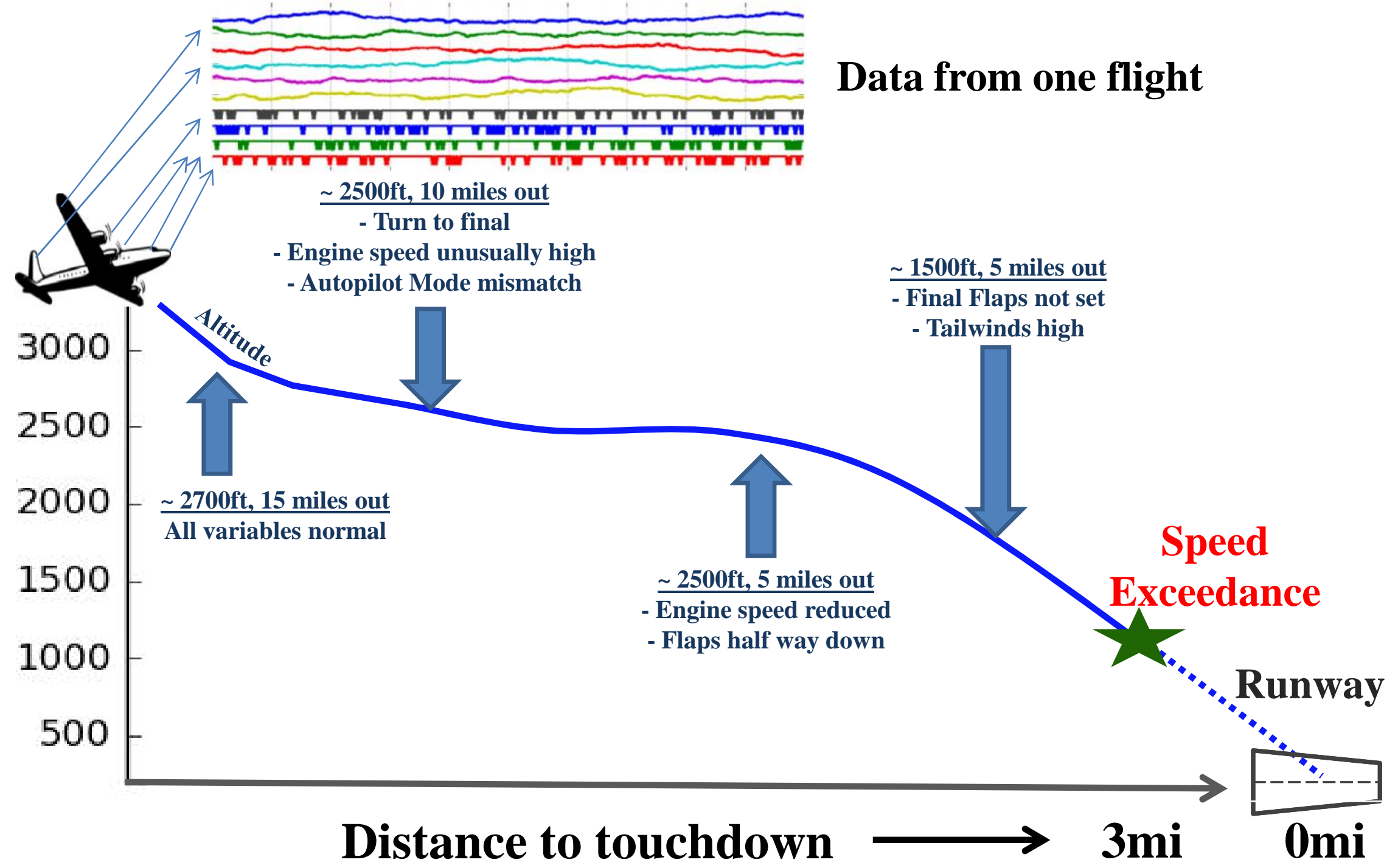




\section{Precursor discovery}

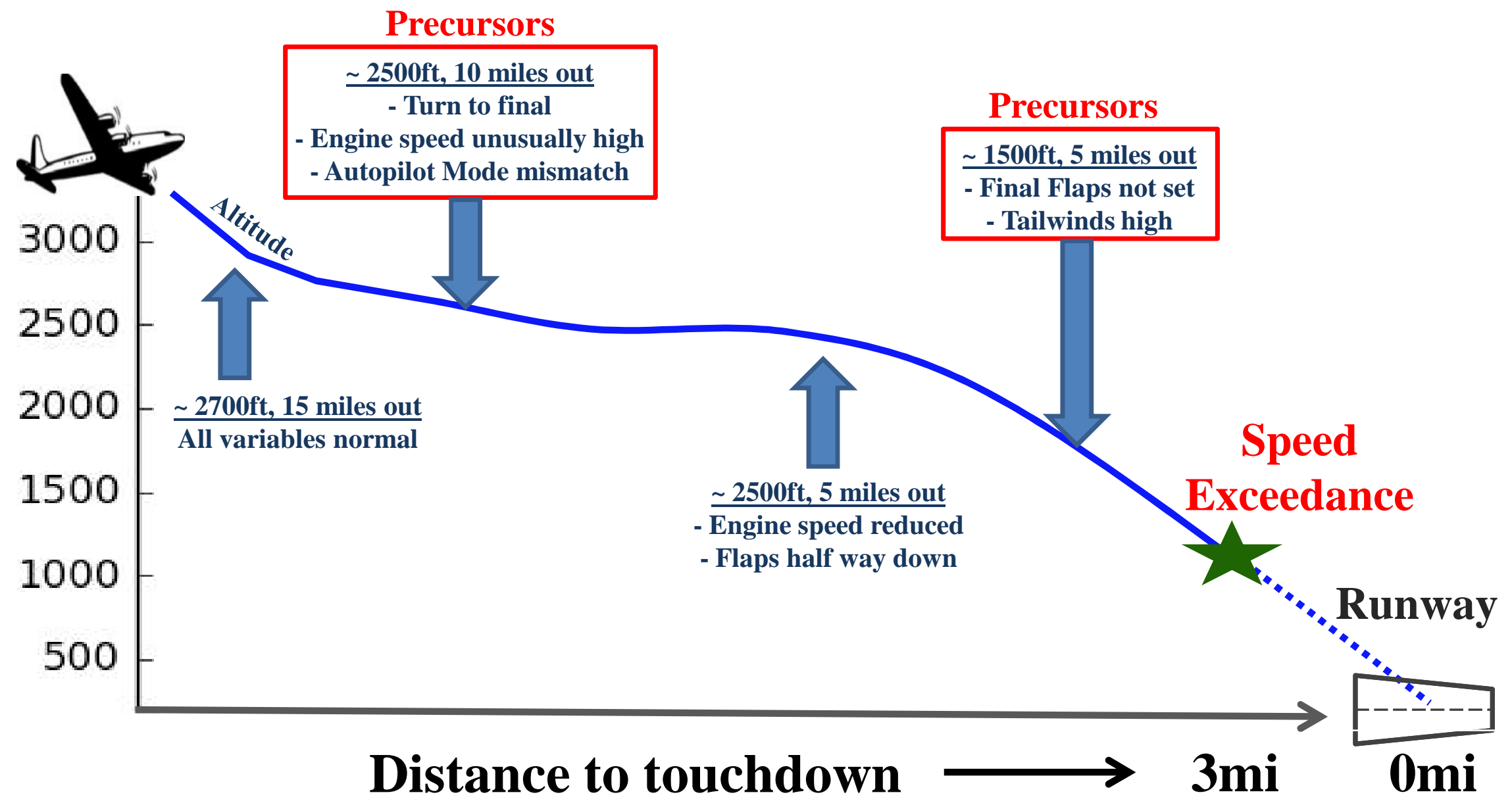




\section{Precursor discovery}

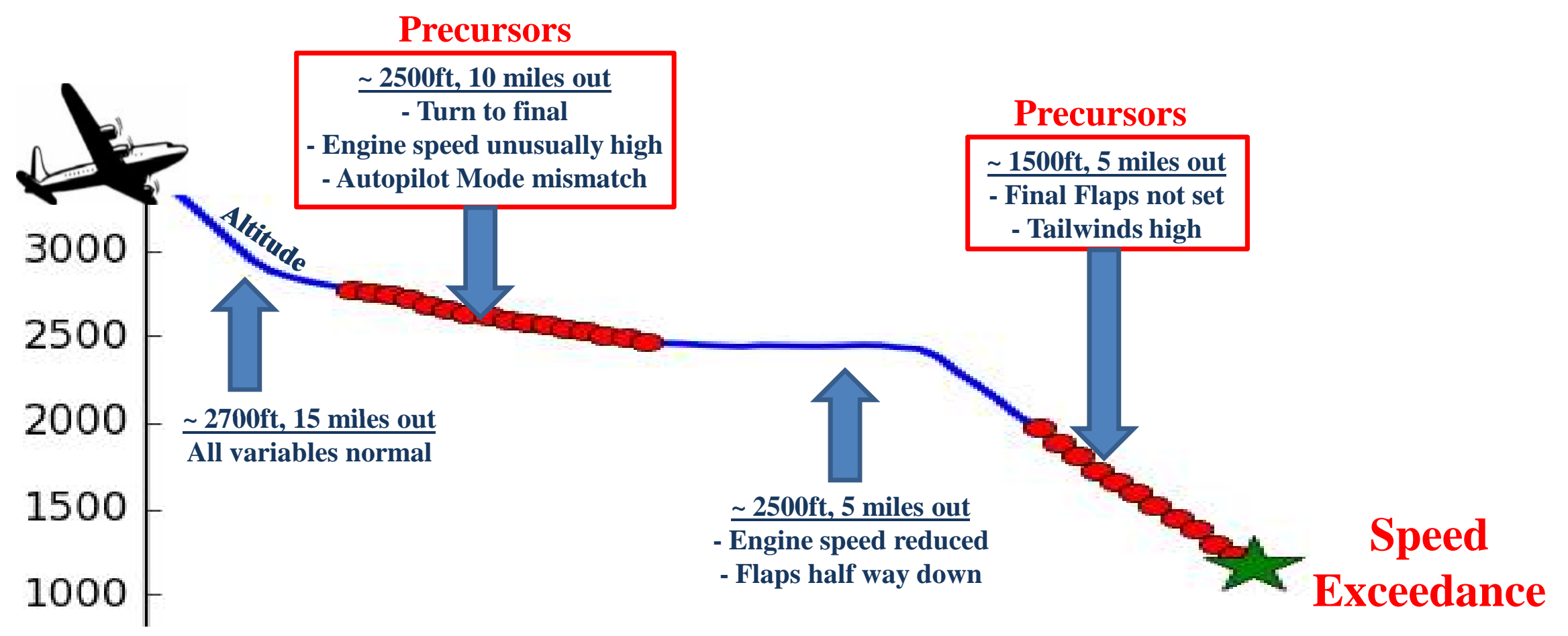

Probability of speed exceedance

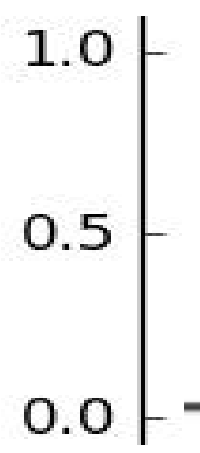

Flight timeline 


\section{Precursor discovery}

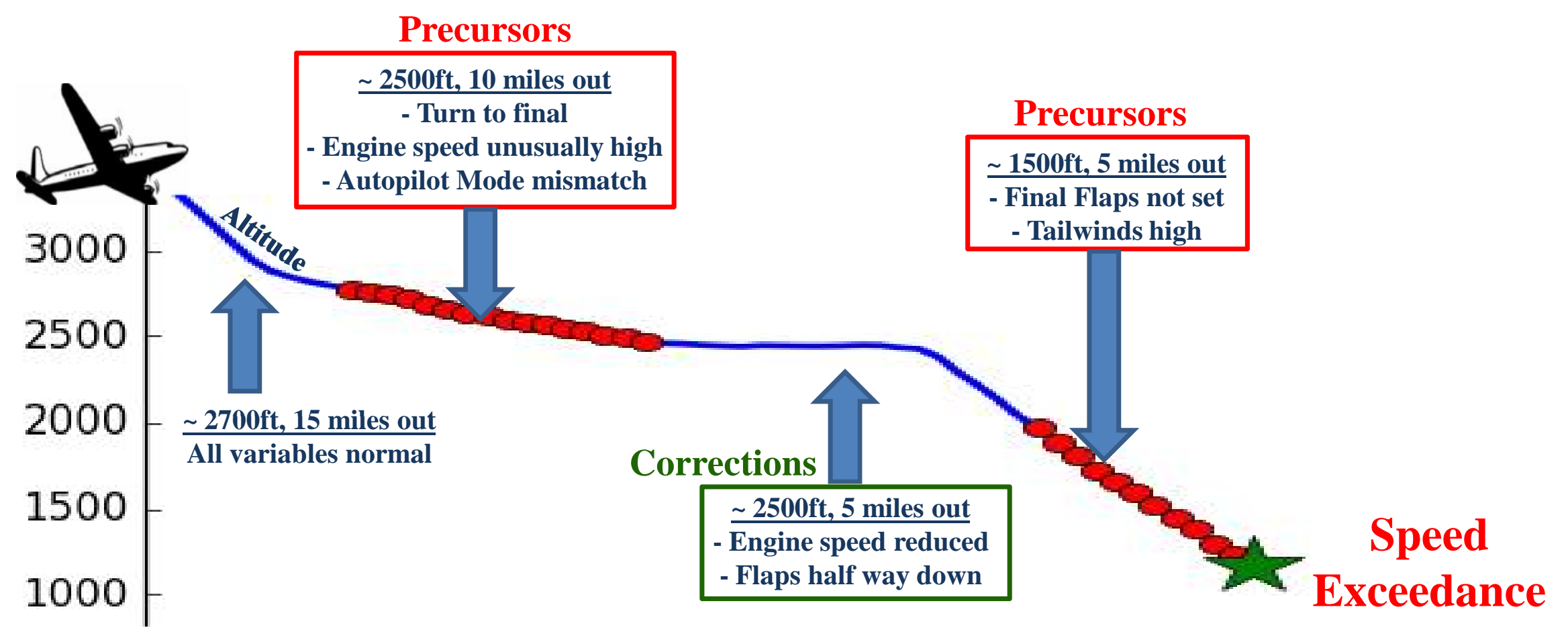

Probability of speed exceedance

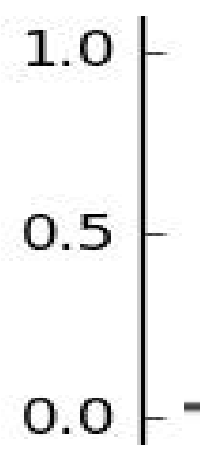

Flight timeline 


\section{Precursor discovery}

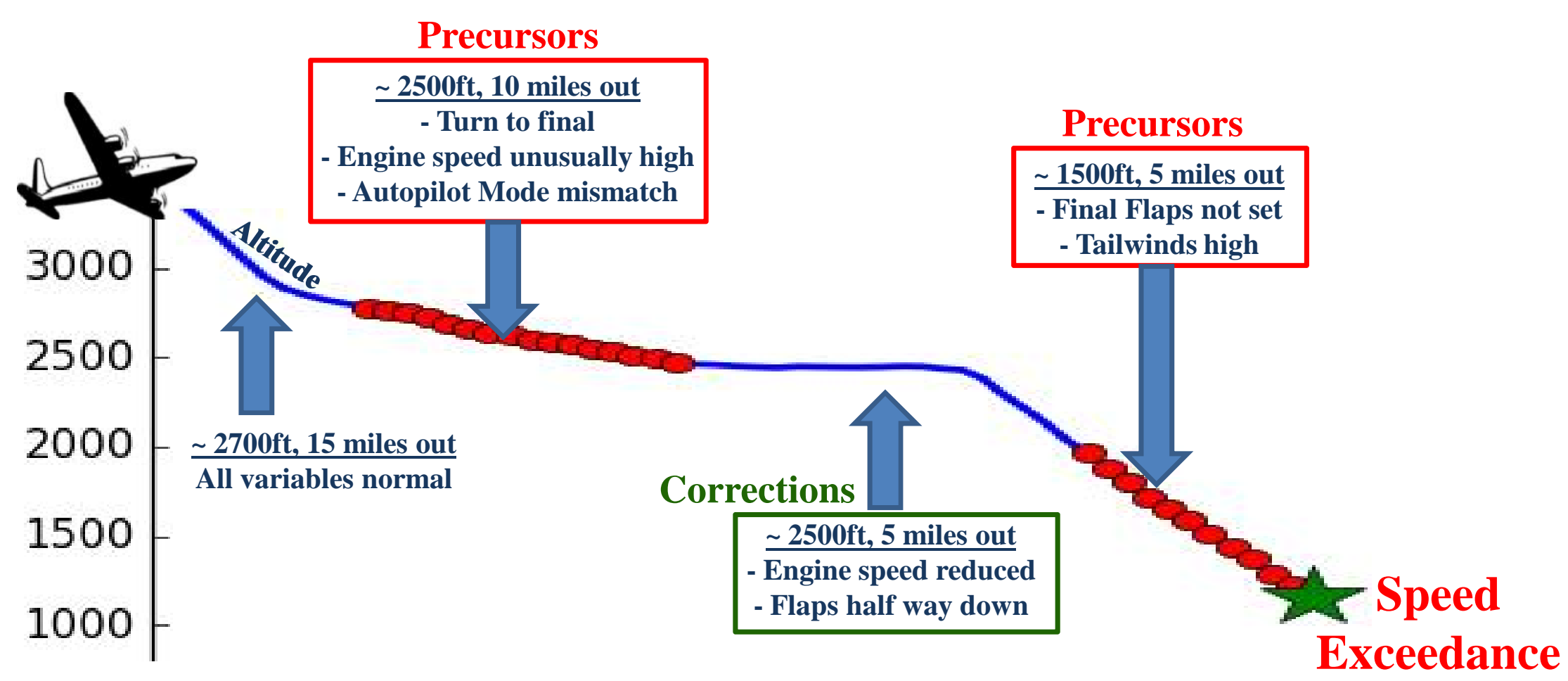

\section{Adverse event may be any event of interest}

- $\quad$ Single flight safety events such as exceedances, go-around, stall,

- Multi-flight safety events such as loss of separation, TCAS events,

- $\quad$ Airspace or NAS level events such as GDP, congested sectors, delays,

- Performance events such as high throughput, mission success, 


\section{Why find precursors?}

\section{Forensic analysis of past events}

- Accident investigation

- Hazard identification

- Operations

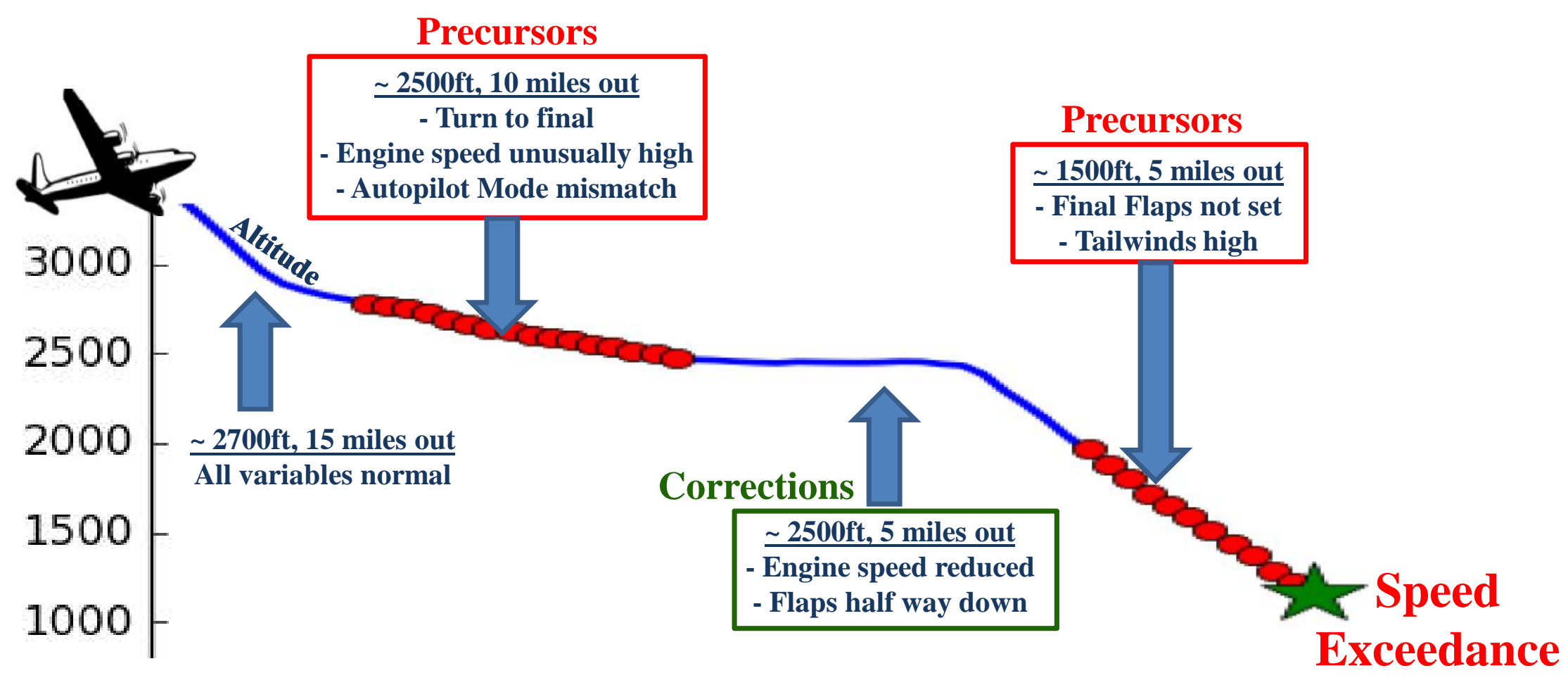




\section{Why find precursors?}

\section{Real-time decision support}

- Crew alerting, Situational awareness, Action recommendation 


\section{Why find precursors?}

- Forecasting adverse events better and earlier

- Generate a knowledge base (precursors)

- Develop decision support tools

- Alerting systems

- Recommendation systems on corrective actions

- Improve operator training

- Response and recovery from precursors

- Predictive maintenance

- Precursors to component failures 


\section{Challenges in Precursor Discovery}

- Human expert analysis is not scalable

- Not easy to find patterns in 100s of time series.

- Visualization is almost impossible.

- Subjective variations among experts

- Costly and slow

- Data mining is not easy

- High dimensions (100s of variables)

- High velocity of data (1000s of flights per day)

- Data heterogeneity (continuous, categorical, text, voice, video)

- Precursors are unlabeled. 


\section{Outline}

- Background

- Precursor discovery problem, uses, challenges

- Methodology

- ADOPT algorithm

- Case Studies

- Take-off Stall Hazard

- STAR procedure adherence

- Summary 


\section{Anatomy of a Safety Event}

Safe

Compromised States

Anomolous

Safe States or Accidents

States

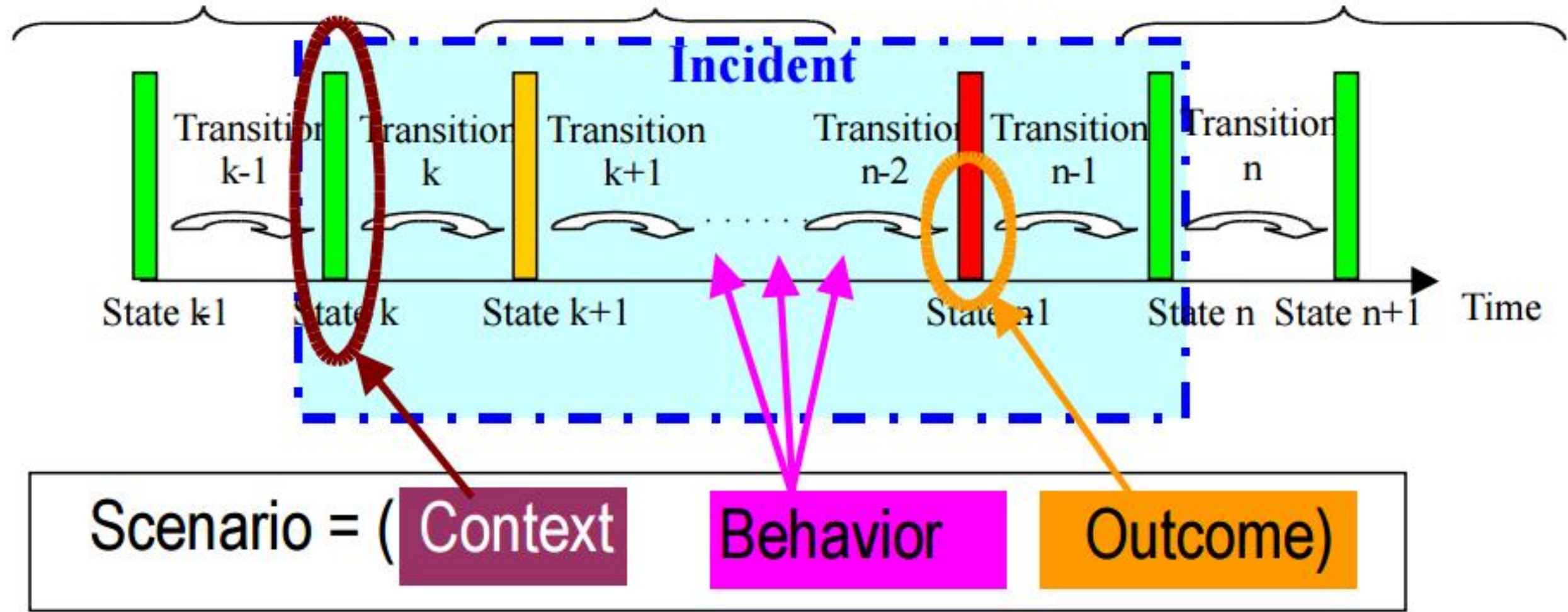




\section{Precursor discovery using data}

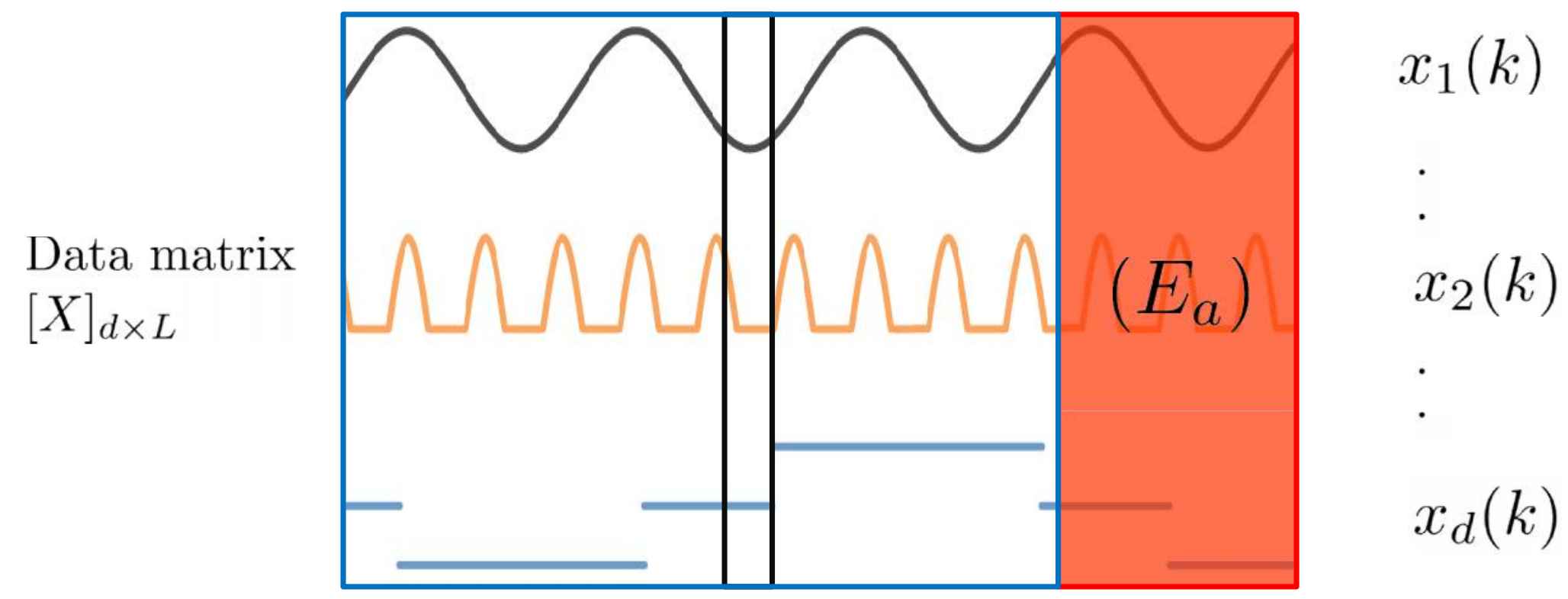

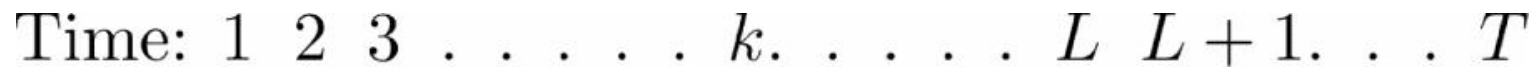

$\left.\begin{array}{c}\text { Event at } \\ \text { time k }\end{array}\left[\begin{array}{c}x_{1}(k) \\ x_{2}(k) \\ \cdot \\ x_{d}(k)\end{array}\right]\right\} \begin{gathered}\text { Start of adverse } \\ \text { event }\end{gathered}$




\section{Problem setup}

- Data

- Adverse time series data $\overline{\mathcal{N}}=\left\{X_{i}\right\}, i=1,2, . . \bar{N}$;

- Nominal time series data $\mathcal{N}=\left\{X_{i}\right\}, i=1,2, . . N$;

- Unsupervised

- Event

- A time slice of data $\left[\begin{array}{c}x_{2}(k) \\ \cdot \\ x_{d}(k)\end{array}\right]$

- Data is a sequence of events $X_{i}=\left[\mathbf{x}(\mathbf{1}), \mathbf{x}(\mathbf{2}), . ., \mathbf{x}\left(\mathbf{L}_{\mathbf{i}}\right)\right]_{i}$ 


\section{Precursor Definition}

Given a sequence of events $X=[\mathbf{x}(\mathbf{1}), \mathbf{x}(\mathbf{2}), . ., \mathbf{x}(\mathbf{L})]$, an action is any state transition $a_{k}: \mathbf{x}(\mathbf{k}) \longrightarrow \mathbf{x}(\mathbf{k}+\mathbf{1})$ where $1 \leq k \leq L$, then $a_{k}$ is a precursor to $E_{A}$ if

$$
V\left(a_{k}\right)-V\left(a_{k}^{*}\right)>\delta .
$$

where $\delta>0$,

$a_{k}^{*}$ is the expert's action at $k$,

$V(z)$ is the value function $\propto P\left(E_{A} \mid z\right)$ 


\section{Related work}

- Precursor discovery in multivariate time series is a new problem

- No direct algorithm exists

\section{Challenges}

1. Unsupervised (no ground truth on precursors)

2. Temporal (long sequences make it hard)

3. High dimensionality

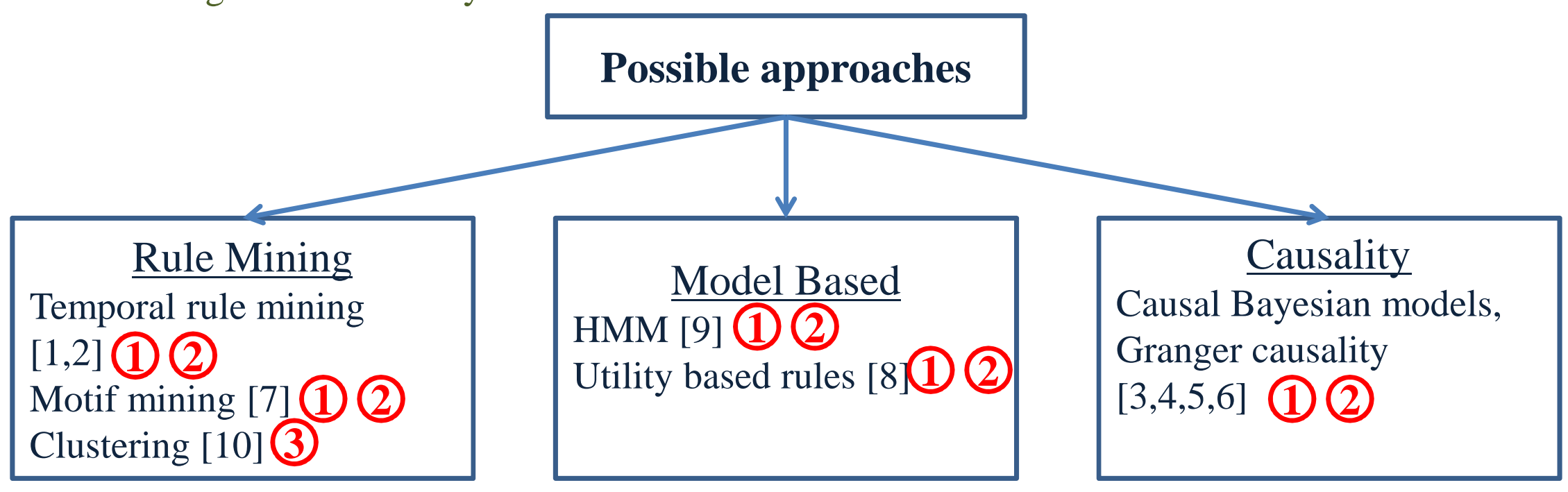

Issues/Drawbacks

(1) Computationally expensive (scales combinatorial/exponential with number of items).

2. Doesn't handle continuous data (or needs discretization which grows combinatorial).

(3) Similarity metric not easy to define for high dimensional data. 


\section{References}

- [1] R. Agrawal and R. Srikant, "Mining sequential patterns," in Proceedings of the Eleventh International Conference on Data Engineering, ser. ICDE '95. Washington, DC, USA: IEEE Computer Society, 1995, pp. 314.

- $\quad$ [2] G. Das, K. ip Lin, H. Mannila, G. Renganathan, and P. Smyth, "Rule discovery from time series," in KDD 98. AAAI Press, 1998, pp. 16-22.

- [3] K. Karimi and H. Hamilton, "Discovering tempo ral/causal rules: A comparison of methods," in Advances in Artificial Intelligence, ser. Lecture Notes in Computer Science, Y. Xiang and B. Chaib-draa, Eds. Springer Berlin Heidelberg, 2003, vol. 2671, pp. 175-189.

- [4] S. Kleinberg, "Causal inference with rare events in large-scale time-series data," in Proceedings of the Twenty-Third International Joint Conference on Artificial Intelligence, ser. IJCAI '13. AAAI Press, 2013, pp. 1444-1450.

- [5] A. Arnold, Y. Liu, and N. Abe, "Temporal causal modeling with graphical granger methods," in Proceedings of the 13th ACM SIGKDD International Conference on Knowledge Discovery and Data Mining, ser. KDD'07. New York, NY, USA: ACM, 2007, pp. 66-75.

- [6] J. B. D. Cabrera and R. K. Mehra, "Extracting Precursor Rules from Time Series: A Classical Statistical Viewpoint," in SIAM International Conference on Data Mining, 2002.

- [7] A. McGovern, D. Rosendahl, R. Brown, and K. Droegemeier, "Identifying predictive multidimensional time series motifs: an application to severe weather prediction," Data Mining and Knowledge Discovery, vol. 22, no. 1-2, pp. 232\{258, 2011.

- [8] G. Maragatham and M. Lakshmi, "A strategy for mining utility based temporal association rules," Trendz in Information Sciences \& Computing (TISC), 2010, Chennai, 2010, pp. 38-41.

- [9] Z. Zhu and G. Deng, "Mining Interest Association Rules in Website Based on Hidden Markov Model," Wireless Communications, Networking and Mobile Computing, 2008. WiCOM '08. 4th International Conference on, Dalian, 2008, pp. 1-4.

- [10] Rani, Y. Leela Sandhya, P. Naga Deepthi, and Ch Rama Devi. "Clustering Algorithm for Temporal Data 


\section{Background: Markov Model}

An MDP is a tuple $\left(\mathcal{S}, \mathcal{A}, P_{s, a}, \gamma, R\right)$

- $\mathcal{S}=\mathbb{R}^{d}$ is a continuous state space with $d$ state variables,

- $\mathcal{A}=\mathbb{R}^{l}$ is an action space with $l$ action variables,

- $\left\{P_{s, s^{\prime}}^{a}\right\}$ (or $P_{s s^{\prime}}$ if actions are unknown) are the state transition probabilities,

- $\gamma \in[0,1]$ is the discount factor,

- $R: \mathcal{S} \rightarrow \mathbb{R}$ is the underlying reward function,

policy: $\pi(s, a)=p(a \mid s)$,

optimal policy: $\pi_{E}(s)=a^{*}$, 


\section{Background: Value Function and Bellman's Optimality}

- The value of state $\mathbf{x}_{\mathbf{0}}$ under policy $\pi$ is $V^{\pi}\left(\mathbf{x}_{\mathbf{0}}\right)=E\left[R\left(\mathbf{x}_{\mathbf{0}}\right)+\gamma R\left(\mathbf{x}_{\mathbf{1}}\right)+. .+\gamma^{L} R\left(\mathbf{x}_{\mathbf{L}}\right) \mid \pi\right]$ where the expectation is over the distribution of sequences starting from $\mathbf{x}_{\mathbf{0}}$.

- The expert's policy $\pi_{E}$ $\pi_{E}(\mathbf{x}) \geq \pi_{i}(\mathbf{x}) \quad \Longleftrightarrow \quad V^{\pi_{E}}(\mathbf{x}) \geq V^{\pi_{i}}(\mathbf{x}) \quad \forall \quad \pi_{i}$

- Bellman's optimality $\pi_{E}\left(\mathbf{x}_{\mathbf{k}}\right)=\arg \max _{\left\{\text {feasible } \mathbf{x}_{\mathbf{k}+\mathbf{1}\}}\right.} V^{\pi_{E}}\left(\mathbf{x}_{\mathbf{k}+\mathbf{1}}\right)$ 


\section{ADOPT Framework}

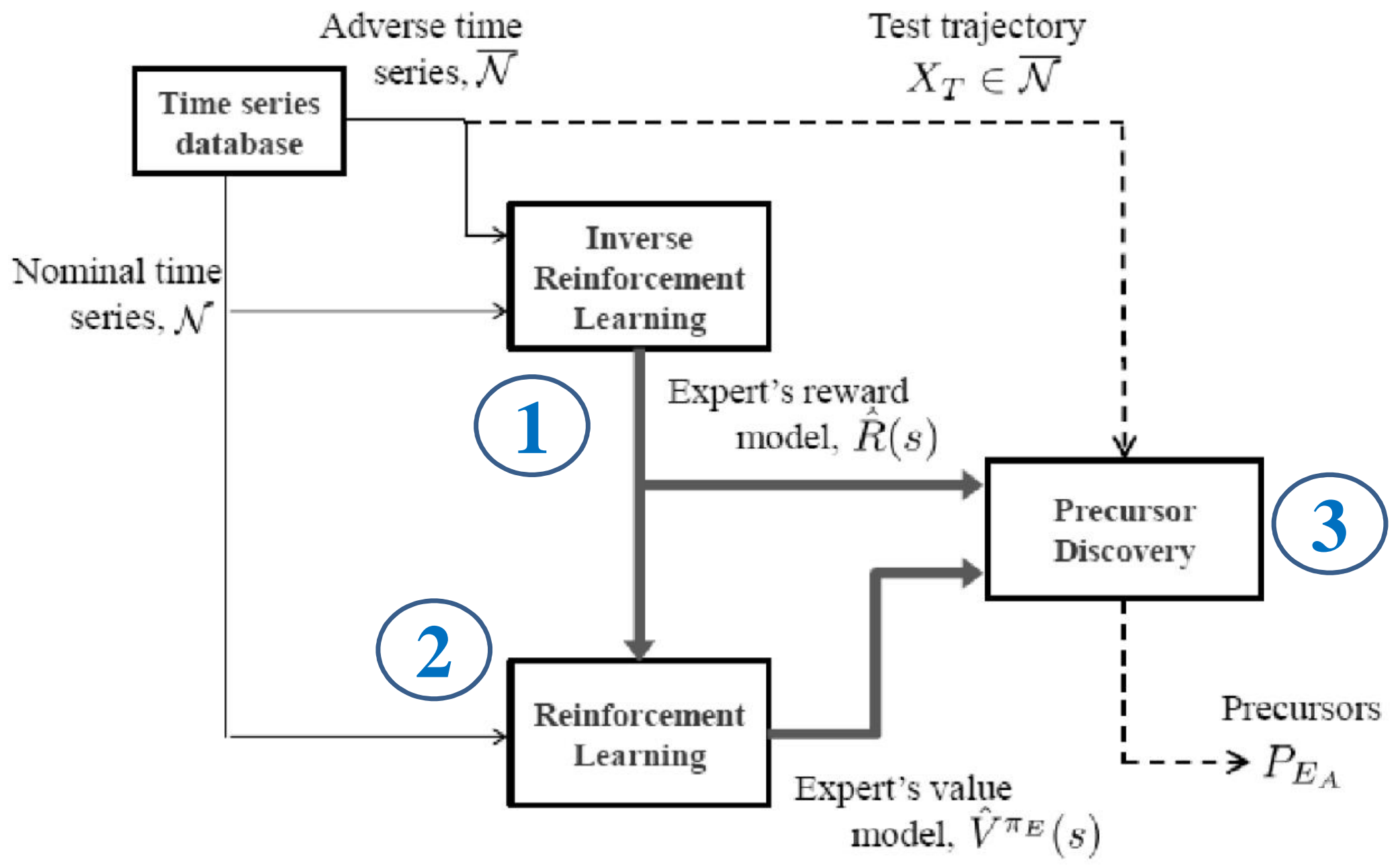




\section{Step 1: Expert's Reward Model}

- $R(\mathbf{x} ; \boldsymbol{\alpha})=\alpha_{1} \phi_{1}(\mathbf{x})+\alpha_{2} \phi_{2}(\mathbf{x})+. .+\alpha_{m} \phi_{m}(\mathbf{x})$

- A general model of the expert's reward

- $\boldsymbol{\alpha}=\left[\begin{array}{llll}\alpha_{1} & \alpha_{2} & \ldots & \alpha_{m}\end{array}\right]^{T}$ to be estimated

- $\phi_{i}(\mathbf{x}) ; i=1,2, . ., m$ are some known basis functions (gaussian)

- $\boldsymbol{\alpha}^{*}=\arg \min _{\boldsymbol{\alpha}}\left\{E_{\mathbf{x}_{0}}\left[V^{\pi_{a d v}}\left(\mathbf{x}_{0} ; \boldsymbol{\alpha}\right)\right]-E_{\mathbf{x}_{0}}\left[V^{\pi_{E}}\left(\mathbf{x}_{0} ; \boldsymbol{\alpha}\right)\right]\right\}$

- such that $\left|\alpha_{i}\right| \leq 1, i=1,1, . ., m \quad \begin{gathered}\text { A.Y. Ng and S. Russell, "Aggorithms for } \\ \text { inverse reinforcement learning," ICML 2000. }\end{gathered}$

$$
\hat{R}(\mathbf{x})=f_{R}\left(\mathbf{x} ; \boldsymbol{\alpha}^{*}\right)=\sum_{i=1}^{m} \alpha_{i}^{*} \phi_{i}(\mathbf{x})
$$




\section{ADOPT Framework}

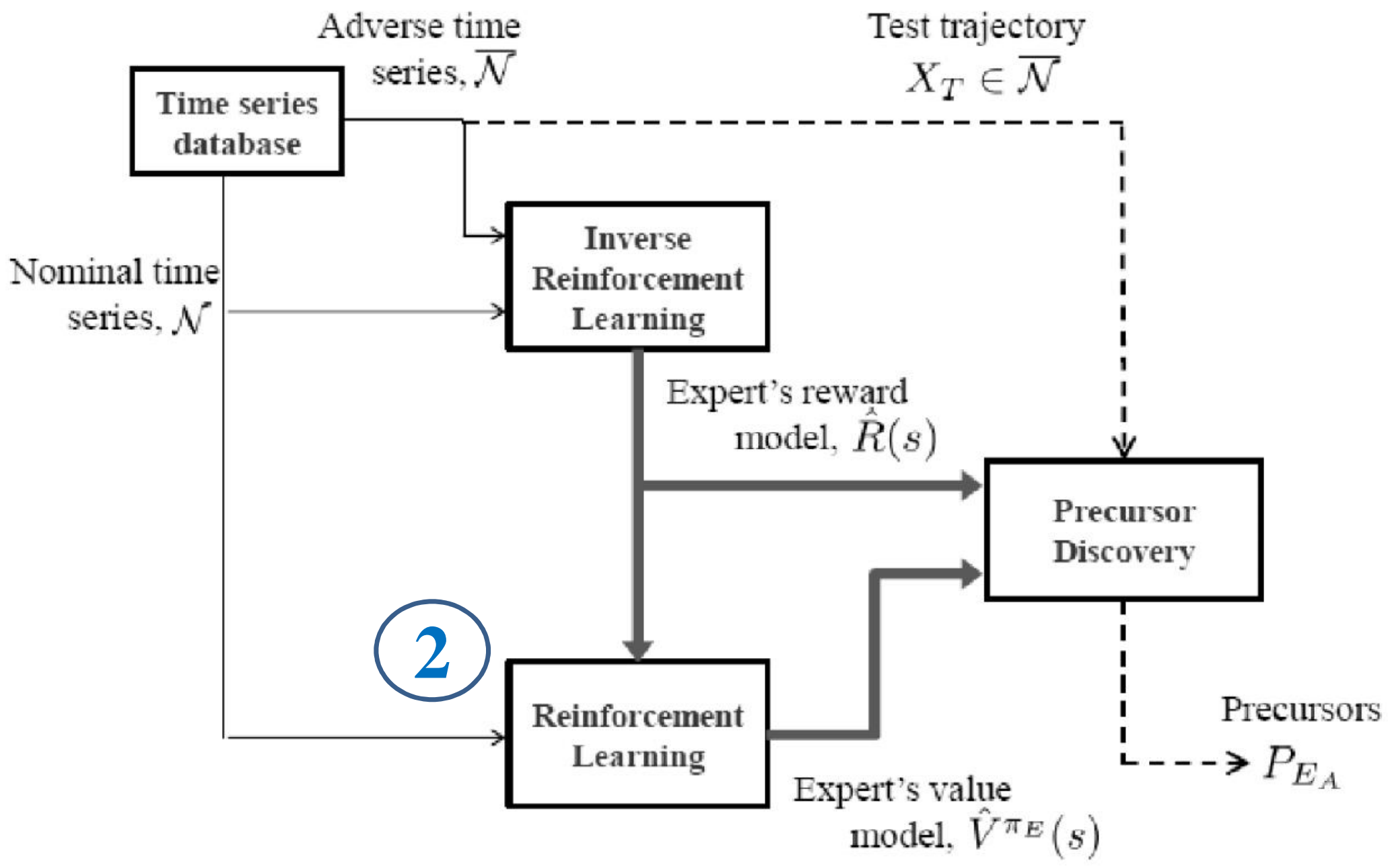




\section{Step 2: Expert's Value Model}

- Value estimation by Monte Carlo method

- Known reward (from previous step)

- Time series data as Monte Carlo samples

- Return $\operatorname{Ret}(\mathbf{x})=\sum \gamma^{k} R\left(\mathbf{x}_{\mathbf{k}}\right)$ for each state $\mathbf{x}$ as accumulated rewards

- For every labeled pair $\left(\mathbf{x}_{i}, \operatorname{Ret}\left(\mathbf{x}_{i}\right)\right)$, a regression model $\hat{V}^{\pi_{E}}(\mathbf{x} ; \theta)$ parameterized by $\theta$ can be built

$-\theta^{*}=\arg \min _{\theta} \frac{1}{N_{s}} \sum_{i=1}^{N_{s}}\left\|\operatorname{Ret}\left(\mathbf{x}_{i}\right)-\hat{V}^{\pi_{E}}\left(\mathbf{x}_{i} ; \theta\right)\right\|^{2}+\frac{\mu}{2}\|\theta\|^{2}$

$$
\hat{V}^{\pi_{E}}(\mathbf{x})=f_{V}\left(\mathbf{x} ; \theta^{*}\right)
$$




\section{ADOPT Framework}

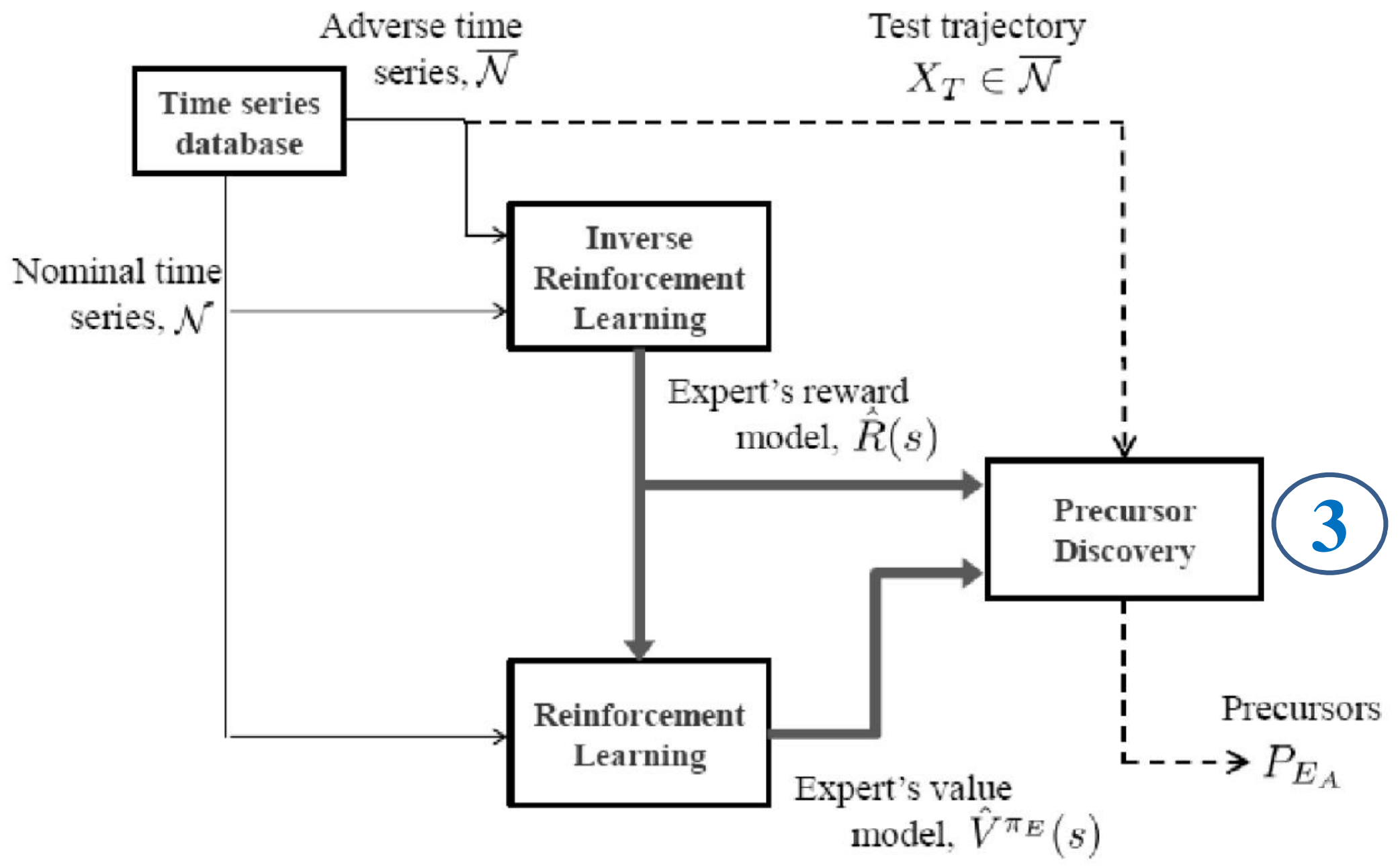




\section{Step 3: Precursor Discovery}

Given a sequence of events $X=[\mathbf{x}(\mathbf{1}), \mathbf{x}(\mathbf{2}), . ., \mathbf{x}(\mathbf{L})]$, an action is any state transition $a_{k}: \mathbf{x}(\mathbf{k}) \longrightarrow \mathbf{x}(\mathbf{k}+\mathbf{1})$ where $1 \leq k \leq L$, then $a_{k}$ is a precursor to $E_{A}$ if

$$
V\left(a_{k}^{*}\right)-V\left(a_{k}\right)>\delta .
$$

- requires finding the "optimal" decision

- Bellman's optimality

$$
\mathbf{x}_{\mathbf{k}+\mathbf{1}}^{*}=\arg \max _{\left\{\text {feasible } \mathbf{x}_{\mathbf{k}+\mathbf{1}}\right\}} V^{\pi_{E}}\left(\mathbf{x}_{\mathbf{k}+\mathbf{1}}\right)
$$

- requires scoring the suboptimal decisions

- $P I_{k}=V^{\pi_{E}}\left(\mathbf{x}_{\mathbf{k}+\mathbf{1}}^{*}\right)-V^{\pi_{E}}\left(\mathbf{x}_{\mathbf{k}+\mathbf{1}}\right)$

- A weighted contribution from reward may be added to tradeoff short term vs long term precursors 


\section{Outline}

- Background

- Precursor discovery problem, uses, challenges

- Methodology

- ADOPT algorithm

- Case Studies

- Take-off Stall Hazard

- STAR procedure adherence

- Summary 


\section{Take-off Stall Hazard}

Adverse event: Drop in airspeed after take-off by at least a 20 knots

Goal: To find precursors using flight recorded data

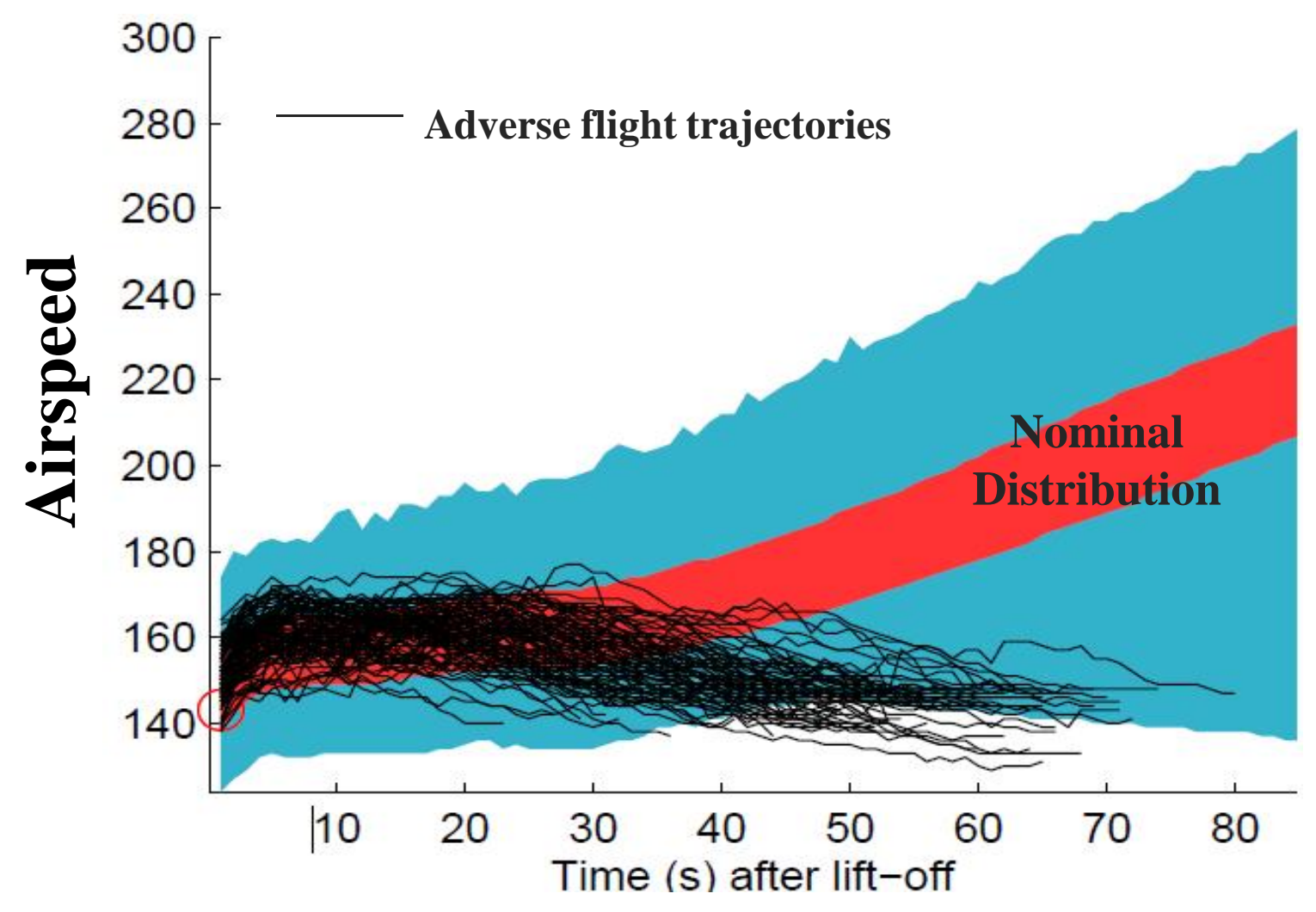




\section{Outline}

- Background

- Precursor discovery problem, uses, challenges

- Methodology

- ADOPT algorithm

- Case Studies

- Take-off Stall Hazard

- Summary 


\section{Factors affecting drop in airspeed}

- Human Factors

- Errors in reference speed calculations, estimating AC weight, energy management.

- human-machine interactions, fatigue, aggressive flying, mode confusion.

- Environmental

- Tail winds, wind shear, sensor failure

- Procedural

- Avoiding terrain, flying over restricted area 


\section{ADOPT analysis}

400 nominal flights

400 adverse flights $200(100+100)$ holdout set

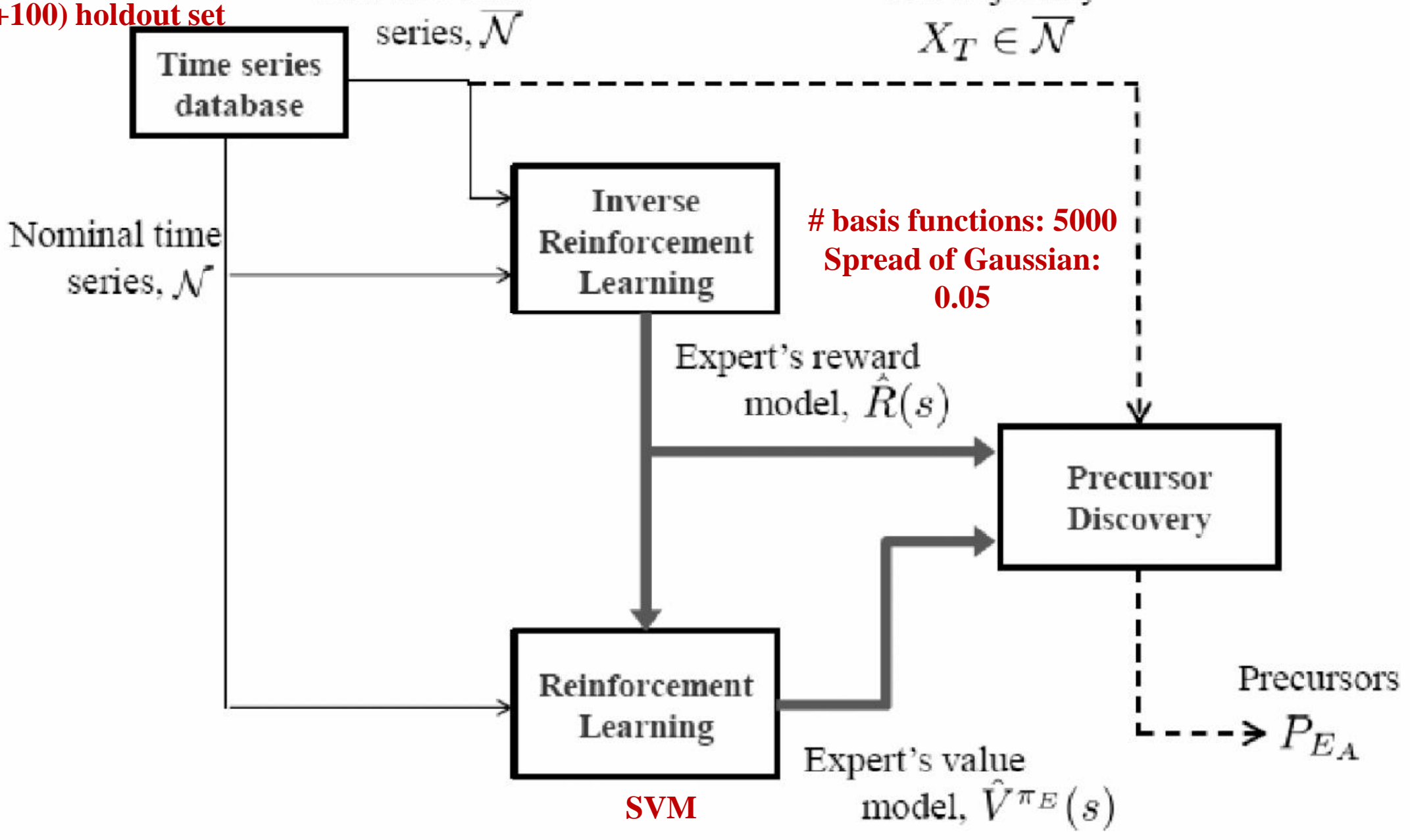




\section{Flight analysis 1 - reference speed set incorrectly}
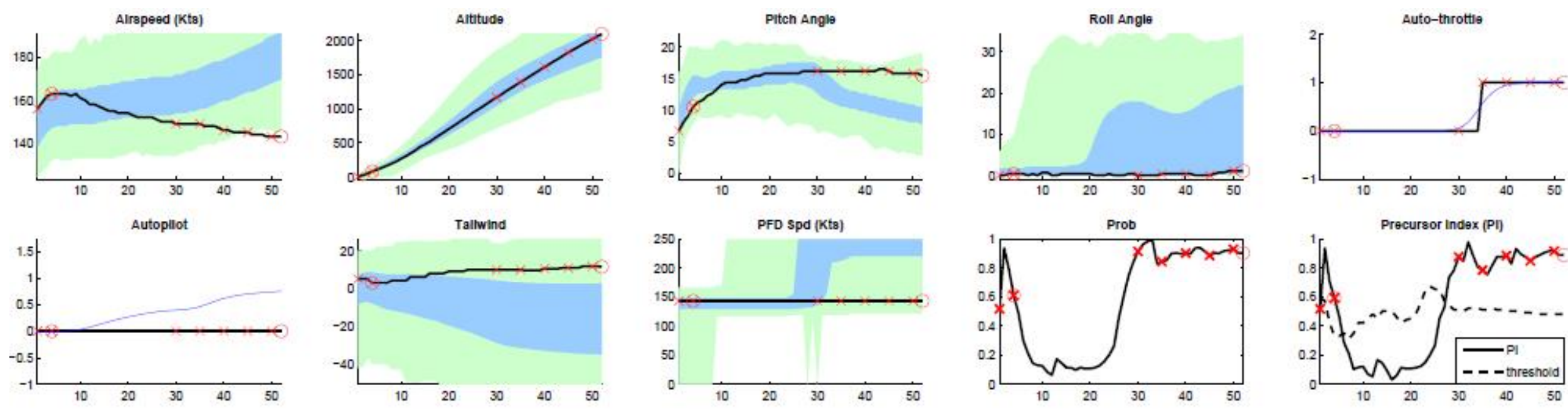

\begin{tabular}{ccccccc}
\hline Time $=1 \mathrm{~s}$ & $4 \mathrm{~s}$ & $30 \mathrm{~s}$ & $35 \mathrm{~s}$ & $40 \mathrm{~s}$ & $45 \mathrm{~s}$ & $50 \mathrm{~s}$ \\
\hline Tailwind & Pitch Angle & PFD Spd & PFD Spd & Pitch Angle & Pitch Angle & PFD Spd \\
Pitch Angle & Altitude & Pitch Angle & Tailwind & PFD Spd & PFD Spd & Pitch Angle \\
Roll Angle & Roll Angle & Auto-throttle & Pitch Angle & Tailwind & Tailwind & Tailwind \\
Altitude & Tailwind & Roll Angle & Roll Angle & Auto-throttle & Auto-throttle & Auto-throttle \\
Auto-throttle & Auto-throttle & Altitude & Altitude & Autopilot & Autopilot & Autopilot \\
\hline
\end{tabular}




\section{Flight analysis 2 - reference speed set incorrectly}
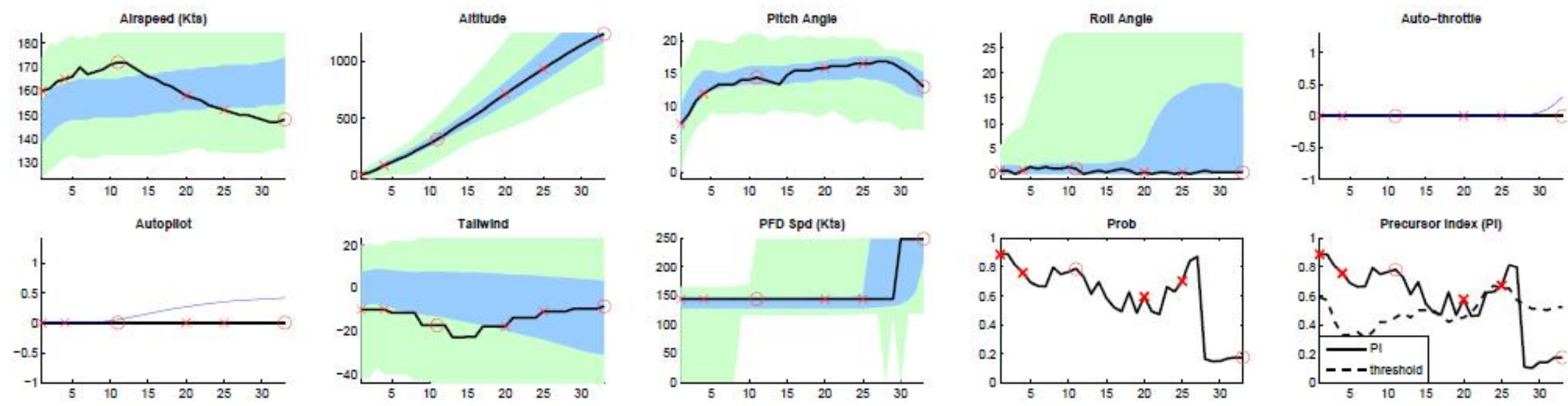

\begin{tabular}{cccc}
\hline Time $=1 \mathrm{~s}$ & $4 \mathrm{~s}$ & $20 \mathrm{~s}$ & $25 \mathrm{~s}$ \\
\hline Pitch Angle & Tailwind & Tailwind & Pitch Angle \\
Tailwind & Pitch Angle & Pitch Angle & PFD Spd \\
Altitude & Roll Angle & Roll Angle & Tailwind \\
Roll Angle & Altitude & PFD Spd & Roll Angle \\
Auto-throttle & Auto-throttle & Altitude & Altitude \\
\hline
\end{tabular}




\section{Flight analysis 1 - Nominal Flight}
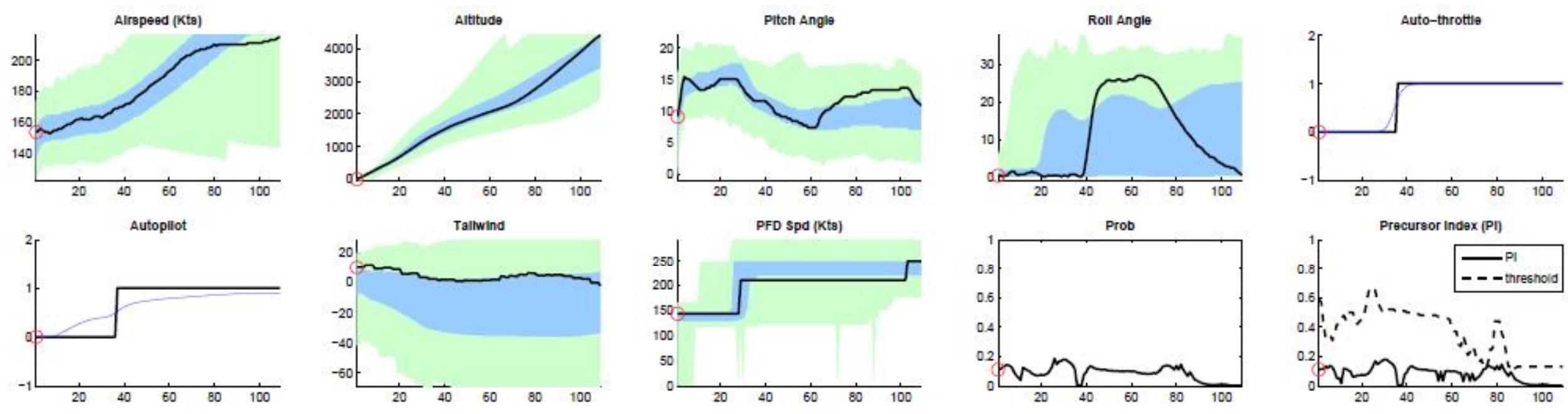


\section{Outline}

- Background

- Precursor discovery problem, uses, challenges

- Methodology

- ADOPT algorithm

- Case Studies

- Take-off Stall Hazard

- STAR procedure adherence

- Summary 


\section{STAR procedure adherence}

Adverse event: Drop in airspeed after take-off by at least a 20 knots

Goal: To find precursors using flight recorded data

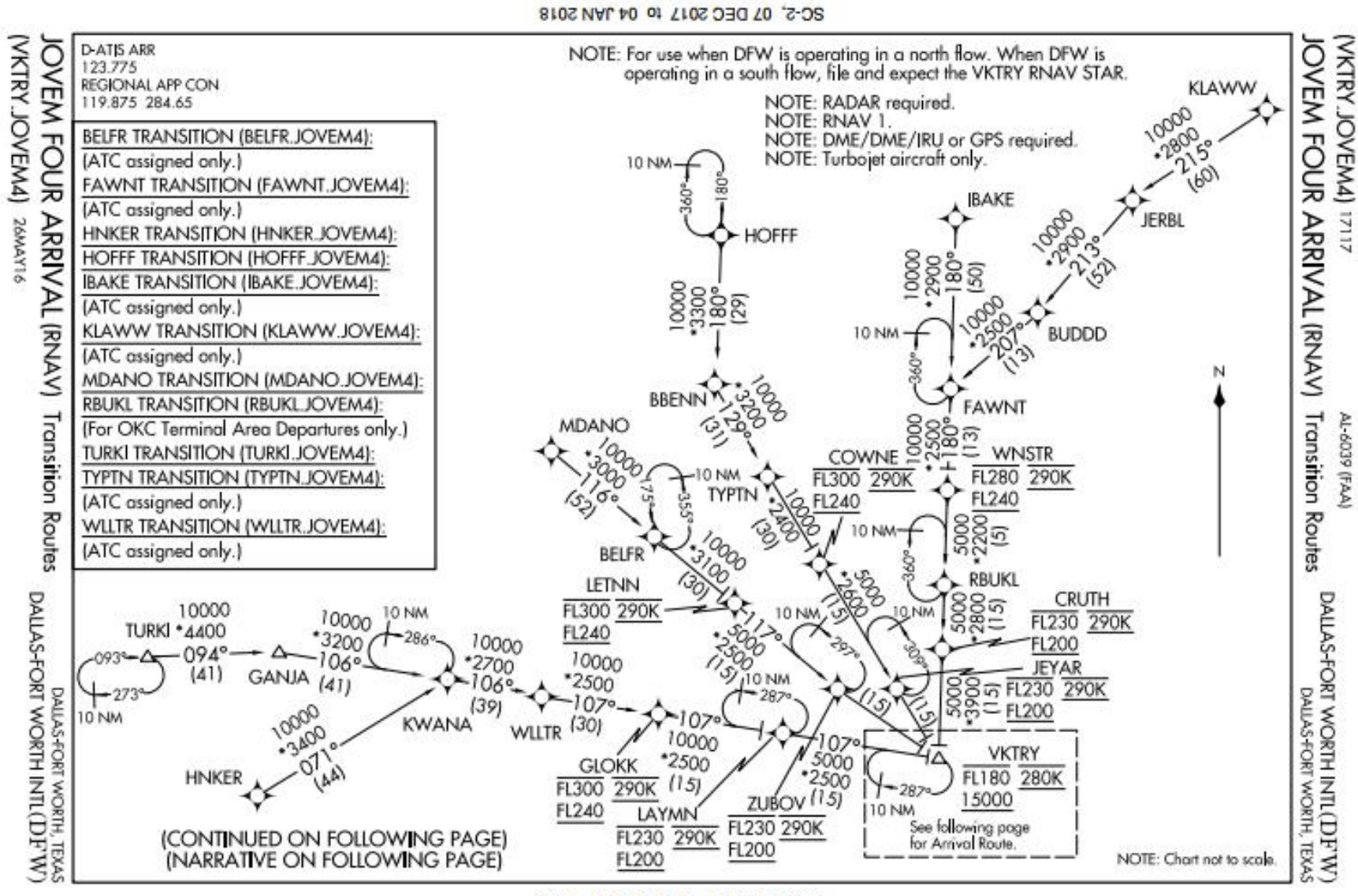

SC-2. 07 DEC 2017 to 04 JAN 2018 


\section{Case Study 2 -STAR procedure adherence}

Adverse event: Drop in airspeed after take-off by at least a 20 knots

Goal: To find precursors using flight recorded data

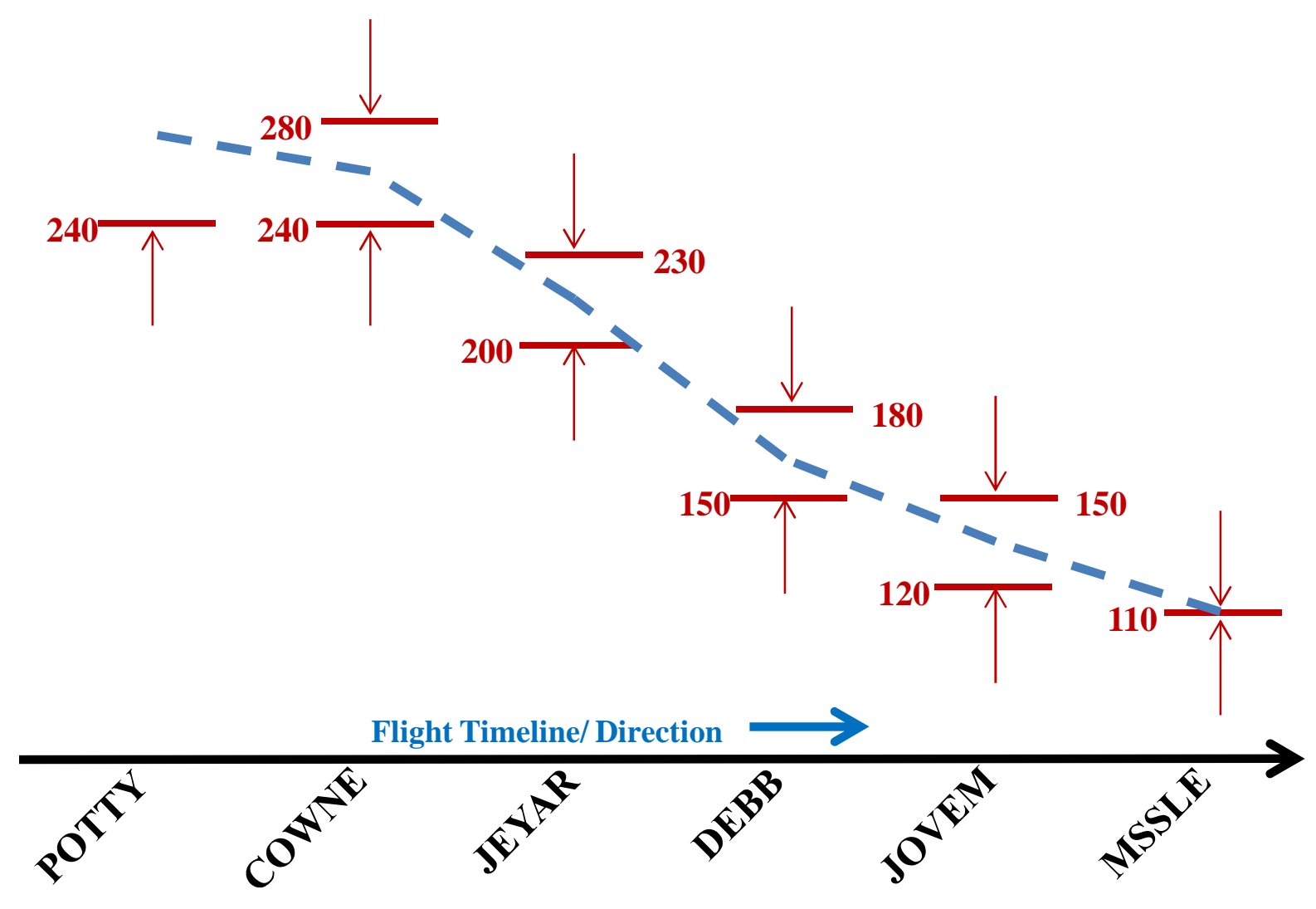




\section{ADOPT analysis}

400 nominal flights

400 adverse flights $200(100+100)$ holdout set

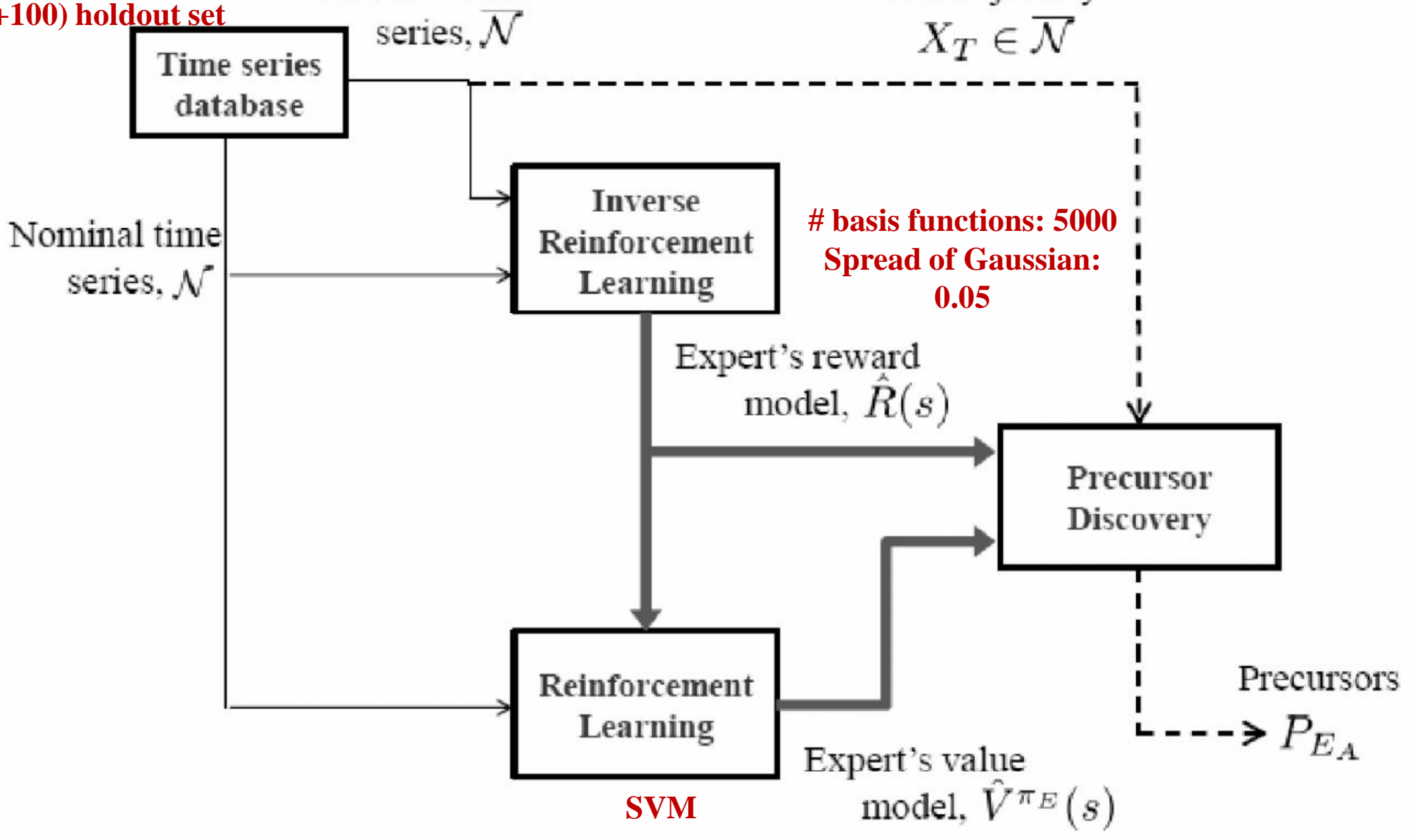




\section{Flight analysis 1 - reference speed set incorrectly}

Required Slope (deg)

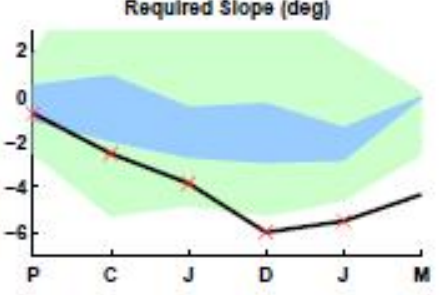

Altitude Sklp

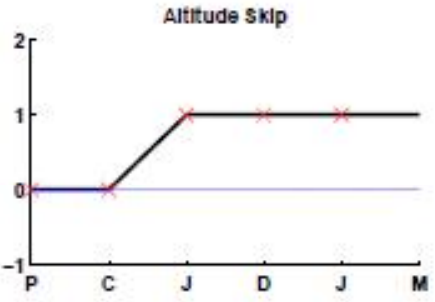

$\times 10^{4} \quad$ Alttude $(\pi)$

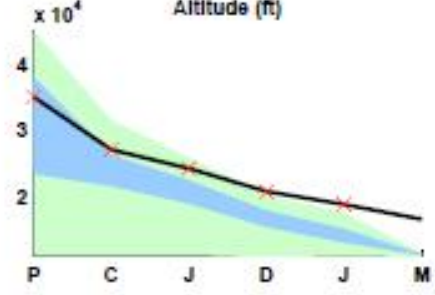

Slope-possible

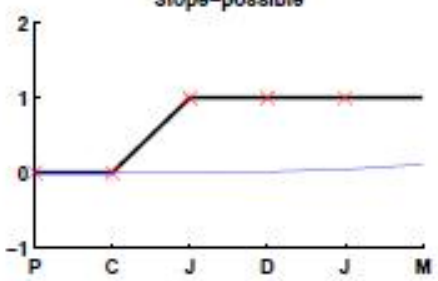

Descent Rate (ti/min)

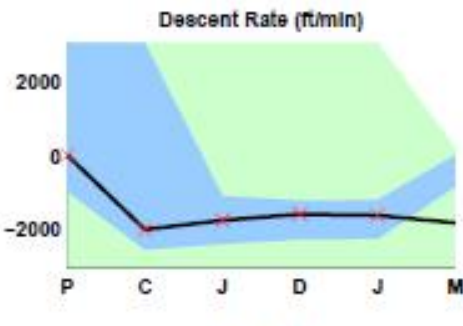

Late-entry

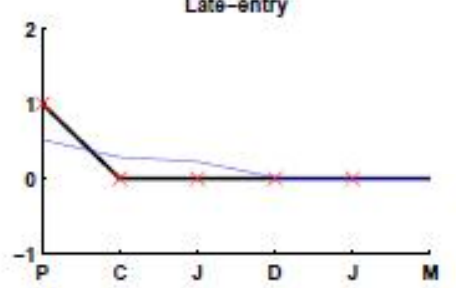

Ground Speed (Kts)
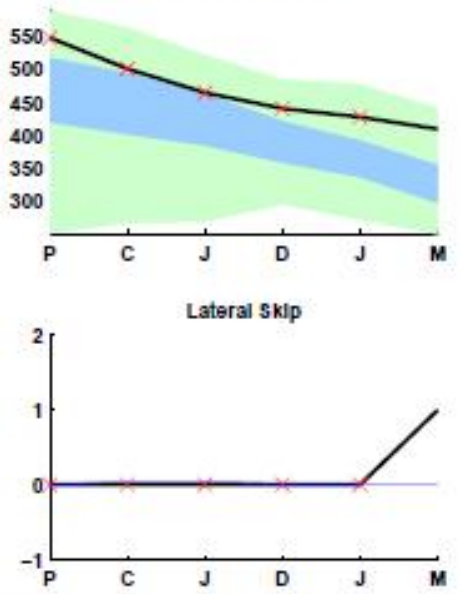

Tall wind (Kts)

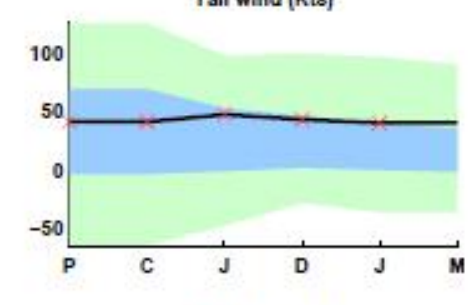

Precursor scor

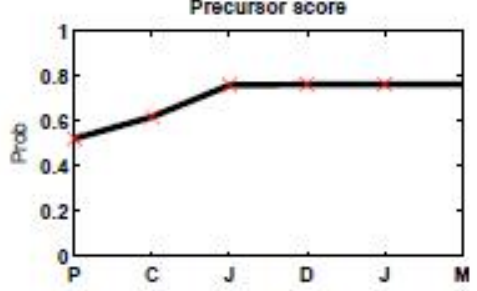

\begin{tabular}{ccccc}
\hline Time $=1 \mathrm{~s}$ & $20 \mathrm{~s}$ & $25 \mathrm{~s}$ & $30 \mathrm{~s}$ & $35 \mathrm{~s}$ \\
\hline Tailwind & Pitch Angle & Pitch Angle & Autopilot & Autopilot \\
Roll Angle & Autopilot & Roll Angle & Roll Angle & Auto-throttle \\
Pitch Angle & Roll Angle & Tailwind & Auto-throttle & Roll Angle \\
Altitude & PFD Spd & PFD Spd & Pitch Angle & Tailwind \\
Auto-throttle & Tailwind & Auto-throttle & Tailwind & Pitch Angle \\
\hline
\end{tabular}




\section{Flight analysis 2 - reference speed set incorrectly}
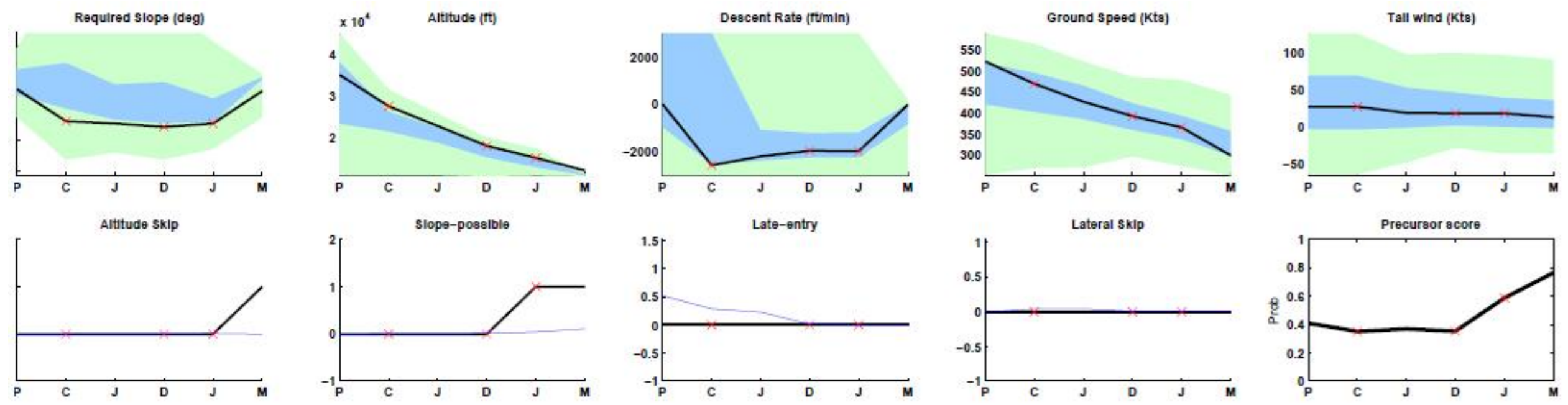

\begin{tabular}{ccccc}
\hline P & C & Je & D & Jo \\
\hline Altitude & Slope-possible & Slope-possible & Slope-possible & Slope-possible \\
Ground Spd & Descent Rate & Ground Spd & Descent Rate & Altitude Skip \\
Descent Rate & Tailwind & Descent Rate & Tailwind & Descent Rate \\
Tailwind & Ground Spd & Tailwind & Ground Spd & Ground Spd \\
Altitude Skip & Altitude & Altitude & Altitude & Tailwind \\
Slope-possible & Altitude Skip & Altitude Skip & Altitude Skip & Altitude \\
\hline
\end{tabular}




\section{Flight analysis 1 - Nominal Flight}

Required Slope (deg)
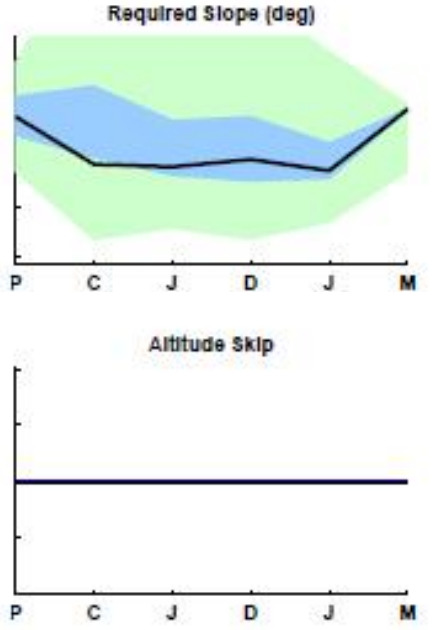

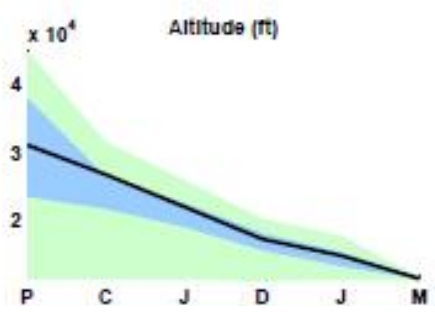

slope-possible

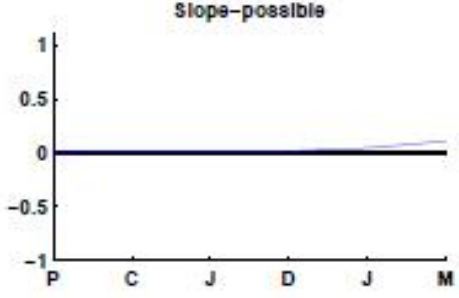

Descent Rate (frumln)
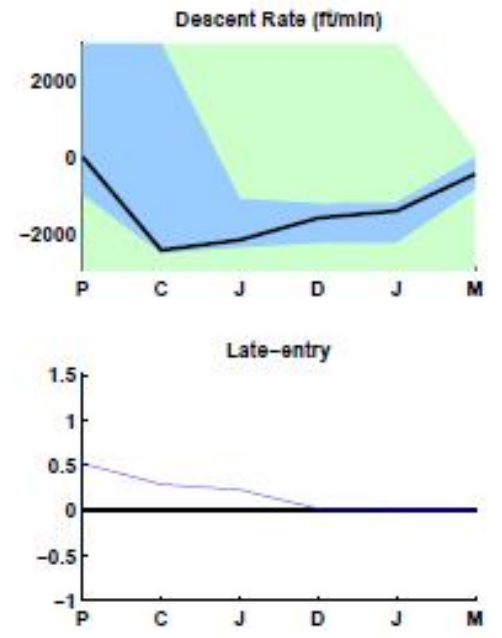

Ground spe日d (Kt8)
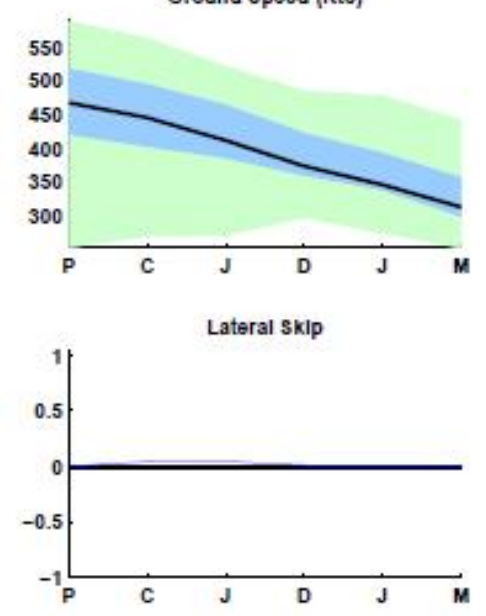

Tall wind (Kta)
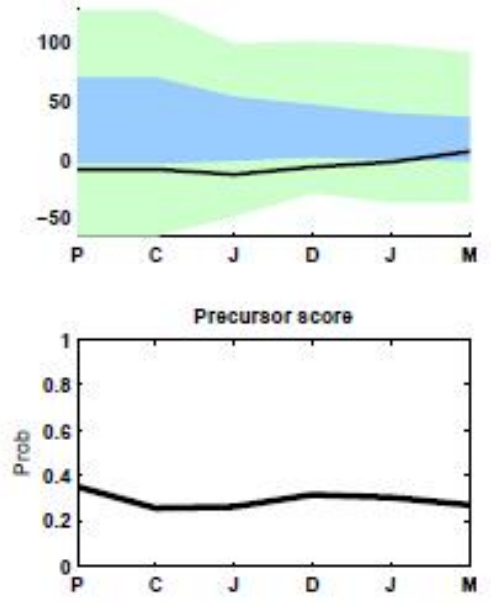


\section{Outline}

- Background

- Precursor discovery problem, uses, challenges

- Methodology

- ADOPT algorithm

- Case Studies

- Take-off Stall Hazard

- STAR procedure adherence

- Summary 


\section{ADOPT's features}

- Data mining based precursor discovery algorithm

- Input

- Feed in time series data with adverse event

- Feed in nominal time series data

- Data could be continuous, categorical, text, images

- Output

- Precursor time instants

- Precursor variables

- Probability score

- Correlation and not Causation 


\section{ADOPT's features}

- Use any/all domain knowledge

- Selecting variables

- Scoping problems in space, time

- Hand-engineering features

- Use any classifier of choice

- SVM, decision tree, K-NN, logistic regression

- Extends to multiple adverse events

- Holistic analysis, safety margins

- Parallelizable

- Multiple CPUs

- Analyze multiple airports, airspaces, aircrafts in parallel 


\section{Summary}

- Precursor discovery is an important problem with uses in multiple applications in Aviation.

- ADOPT is an efficient data mining solution to find precursors.

- Two case studies are presented to show the setup, working and features of ADOPT.

- ADOPT will be open-sourced in the near future. 
Thank You 